\title{
Randomized Trial on the Effects of Attentional Focus on Motor Training of the Upper Extremity Using Robotics With Individuals After Chronic Stroke
}

\author{
Grace J. Kim, PhD, OTR/L, ${ }^{a}$ Jim Hinojosa, PhD, OTR, FAOTA, \\ Ashwini K. Rao, EdD, OTR, FAOTA, ${ }^{c}$ Mitchell Batavia, PhD, PT, ${ }^{d}$ Michael W. O'Dell, MD $^{\mathrm{e}}$
}

From the ${ }^{a}$ Department of Rehabilitation Medicine, Weill Cornell Medical Center, NewYork Presbyterian Hospital, New York, NY; ${ }^{b}$ Department of Occupational Therapy, Steinhardt School Culture, Education, and Human Development, New York University, New York, NY; ${ }^{c}$ Department of Physical Therapy, Rehabilitation \& Regenerative Medicine, G.H. Sergievsky Center, College of Physicians and Surgeons, Columbia University, New York, NY; ${ }^{d}$ Department of Physical Therapy, Steinhardt School Culture, Education, and Human Development, New York University, New York, NY; and ${ }^{~}$ Clinical Rehabilitation Medicine, Weill Cornell Medicine, New York, NY.

Current affiliation for Kim, Department of Occupational Therapy, Steinhardt School Culture, Education, and Human Development, New York University, New York, NY.

\begin{abstract}
Objective: To compare the long-term effects of external focus (EF) and internal focus (IF) of attention after 4 weeks of arm training.

Design: Randomized, repeated-measures, mixed analysis of variance.

Setting: Outpatient clinic.

Participants: Individuals with stroke and moderate-to-severe arm impairment living in the community $(\mathrm{N}=33$; withdrawals: $\mathrm{n}=3$ ).

Interventions: Four-week arm training protocol on a robotic device (12 sessions).

Main Outcome Measures: Joint independence, Fugl-Meyer Assessment, and Wolf Motor Function Test measured at baseline, discharge, and 4week follow-up.

Results: There were no between-group effects for attentional focus. Participants in both groups improved significantly on all outcome measures from baseline to discharge and maintained those changes at 4-week follow-up regardless of group assignment (joint independence EF condition: $\mathrm{F}_{1.6,45.4}=17.74 ; P<.0005$; partial $\eta^{2}=.39$; joint independence IF condition: $\mathrm{F}_{2,56}=18.66 ; P<.0005$; partial $\eta^{2}=.40$; Fugl-Meyer Assessment: $\mathrm{F}_{2,56}=27.83 ; P<.0005$; partial $\eta^{2}=.50$; Wolf Motor Function Test: $\mathrm{F}_{2,56}=14.05 ; P<.0005$; partial $\eta^{2}=.35$ ).

Conclusions: There were no differences in retention of motor skills between EF and IF participants 4 weeks after arm training, suggesting that individuals with moderate-to-severe arm impairment may not experience the advantages of an EF found in healthy individuals. Attentional focus is most likely not an active ingredient for retention of trained motor skills for individuals with moderate-to-severe arm impairment, whereas dosage and intensity of practice appear to be pivotal. Future studies should investigate the long-term effects of attentional focus for individuals with mild arm impairment.
\end{abstract}

Archives of Physical Medicine and Rehabilitation 2017;98:1924-31

(C) 2017 by the American Congress of Rehabilitation Medicine

Presented as a poster to the American Congress of Rehabilitation Medicine, November 2-3, 2016, Chicago, IL.

Supported by the Mitchell Leaska Dissertation Award, Steinhardt School Culture, Education, and Human Development, New York University.

Clinical Trial Registration No.: NCT02890446.

Disclosures: Kim has been a paid guest speaker at the HOSPI Robotics Training Seminar discussing the clinical application of the InMotion ARM robot in June 2014. The other authors have nothing to disclose.
An audio podcast accompanies this article. Listen at www.archives-pmr.org.

Attention is a type of cognitive effort that refers to the consciousness or awareness needed to carry out an activity. ${ }^{1}$ Because almost everything a person does requires some attention, it is important for clinicians to understand the role attention plays 
during motor training. What we direct our attention to can affect how well we learn new motor skills or relearn lost skills after injury. ${ }^{2}$ Within a motor learning framework, attentional focus is what someone actively thinks about while performing a motor task. ${ }^{3}$ Two common types of attentional focus are internal focus (IF) and external focus (EF). IF directs a person to think about how he or she is actually moving his or her body during a motor task, whereas EF directs a person to think about the result of his or her movement-the end goal. ${ }^{2}$ A clinician can provide specific instructions or cues prior to motor performance to direct a patient to either an IF or an EF during motor tasks.

According to the constrained action hypothesis, adopting EF allows a healthy individual to use automatic motor control processes that naturally occur in the motor system to facilitate motor skill learning, whereas IF disrupts the automaticity of the motor system, impeding maximum motor performance. ${ }^{4-7}$ Research involving healthy individuals widely supports the use of an EF of attention during motor training to facilitate greater retention of motor improvements. ${ }^{4,8-16}$ Furthermore, IF may actually interfere with the acquisition of new motor skills. ${ }^{5,15}$ Few studies ${ }^{17-19}$ have specifically examined the role of attentional focus during motor performance in individuals recovering from stroke. An EF approach has been shown to be more beneficial than an IF approach to improve temporary performance of arm reach in individuals with mild arm impairment after a single training session. ${ }^{17,18}$ No differences were found between an EF and IF approach during performance of lower limb stepping in individuals with moderate leg impairment. ${ }^{19}$ To our knowledge, this is the first study to investigate the effects of attentional focus on the retention of upper extremity motor skills. Based on the constrained action hypothesis ${ }^{5,15}$ and existing attentional focus literature, we hypothesized that the EF group would achieve greater retention in arm ability versus the IF group after completing 12 sessions of robotic arm training.

\section{Methods}

\section{Participants}

Institutional review board approval was obtained from the academic institution in the New York metropolitan area where the study was conducted. All data collection was completed in an outpatient robotics research laboratory in the department of rehabilitation medicine. Prospective participants provided written informed consent at the start of the screening visit. See appendix 1 for complete inclusion/exclusion criteria, table 1 for key characteristics of the participants, and figure 1 for the Consolidated Standards of Reporting Trials flow diagram.

\section{Study design}

This was a randomized experimental study that used a mixedsubject repeated-measures analysis of variance design. Participants were randomly assigned to either an EF or IF group. Group

$$
\begin{aligned}
& \text { List of abbreviations: } \\
& \text { EF external focus } \\
& \text { FMA Fugl-Meyer Assessment } \\
& \text { IF internal focus } \\
& \text { WMFT Wolf Motor Function Test }
\end{aligned}
$$

assignment was the between-group factor, and time was the within-group factor for primary outcome measures. A block randomization strategy was implemented using SPSS-generated random number sequences. ${ }^{a}$ A member of the research team not involved with the study treatment or outcome measurement implemented the block randomization and allocated subjects to group assignment based on their level of arm impairment and order of acceptance into the study. Treatment dose was defined as the number of repetitions completed, rather than treatment time, because level of impairment could affect the time needed to complete training. All data were analyzed using SPSS Version 23. The effect of arm training was measured by joint independence, Fugl-Meyer Assessment (FMA), and Wolf Motor Function Test (WMFT) from baseline to discharge; retention was measured by the same outcomes from discharge to 4-week follow-up.

\section{Arm training}

Participants in both groups trained using the adaptive mode of the InMotion ARM, ${ }^{\mathrm{b}}$ which standardized arm training to a clock pattern on a horizontal plane. The robot provided physical assistance if participants did not initiate movement in a certain amount of time or veered away from a straight path. Training on the InMotion ARM allowed individuals with moderate-to-severe arm impairment to participate in highly intensive arm therapy while minimizing frustration. Participants in both groups practiced the same movement patterns throughout the protocol. However, the practice condition and the verbal instructions for each group were different to better isolate the assigned attentional focus.

\section{EF group}

The EF group was directed to focus their attention at a video monitor and to move a yellow ball to various targets on the clock design. The affected arm was occluded from view during training to encourage attention to the goal of the video game instead of the moving limb. See figure $2 \mathrm{~A}$ for the $\mathrm{EF}$ practice condition.

\section{IF group}

Participants practiced the same arm training protocol, but the video monitor was turned off to eliminate the task goal and direct attention to the movement of their affected arm during training. See figure $2 \mathrm{~B}$ for the IF practice condition.

Participants received EF- or IF-specific verbal instructions at the start of the study and subsequently during regular breaks every 80 repetitions throughout the training. Everyone practiced the same arm movement pattern (fig 2C) regardless of group assignment throughout the study. The InMotion ARM provided performance feedback scores to both groups every 80 repetitions on the amount of assistance provided, rate of initiation, and distance from the target. The haptic and performance feedback helped both groups refine their movement patterns. Both groups were assigned equivalent doses: 960 repetitions per treatment over 12 sessions. All participants wore a seat belt with shoulder/hip straps to promote neutral trunk alignment and minimize compensatory forward trunk movement during reaching.

\section{Primary outcome measures}

Kinematic and clinical outcomes were measured at baseline, discharge, and 4-week follow-up. Joint independence, a kinematic 
Table 1 Demographic and dosage data $(n=30)$

\begin{tabular}{llll}
\hline Characteristic & EF Group & IF Group & Total \\
\hline Age (y) & $57.3 \pm 16.7$ & $58.9 \pm 8.1$ & $58.1 \pm 12.6$ \\
Chronicity (y) & $5.4 \pm 3.2$ & $4.7 \pm 3.7$ & $5.1 \pm 3.4$ \\
Female (male) & $8 \pm 7$ & $8 \pm 7$ & $16 \pm 14$ \\
Dosage* & $935.1 \pm 37.8$ & $883.8 \pm 74.3$ & $909.5 \pm 63.5$ \\
Stroke type & & & \\
$\quad$ Ischemic & 11 & 9 & 20 \\
$\quad$ Hemorrhagic & 3 & 3 & 6 \\
$\quad$ Both & 1 & 1 & 2 \\
$\quad$ Missing & 0 & 2 & 2 \\
Arm impairment & & & \\
$\quad$ Moderate & 7 & 8 & 15 \\
$\quad$ Severe & 8 & 7 & 15 \\
\hline
\end{tabular}

NOTE. Values are mean \pm SD or number of participants. There were no significant differences between the 2 treatment groups for age and chronicity at baseline.

Abbreviation: Dosage, average number of repetitions completed per participant per treatment session, 960 repetitions was maximum.

* Participants in the EF group completed significantly more repetitions per session than participants in the IF group $\left(t_{28}=2.39\right.$, $P=.024)$.

parameter assessed by a circle drawing task on the InMotion ARM, provides precise objective data with a resolution of $0.1 \mathrm{~mm}$ for position ${ }^{20,21}$ and is predictive of upper extremity clinical measures such the FMA. ${ }^{21}$ It measures the joint angle correlation between $\geq 2$ joints and indicates the degree of coordination between multiple joints. ${ }^{21}$ A decrease in the correlation between 2 joints indicates more independent movement and better motor control of individual joints. ${ }^{22}$ To rule out any testing effects during the assessment, ${ }^{10}$ joint independence was assessed under both the EF training and IF training conditions.

The FMA upper extremity subscale is widely used in research and clinical settings to measure change in motor impairment after acute and chronic stroke. ${ }^{23}$ It has excellent inter- and intrarater reliability and construct validity. ${ }^{23}$ Scoring is based on a 3-point ordinal scale from 0 to 2, and a total score of 66 is determined from the sum of all items. ${ }^{24}$ In our study, we used a total score of 60 because 6 points measure the presence of reflexes, which is considered a separate construct. $^{25}$ Moderate and severe arm impairment were defined by FMA scores of 20 to 47 and 1 to 19 , respectively. ${ }^{25}$

The WMFT consists of 15 performance-based items and 2 strength items, reflecting impairment and activity-level tasks. ${ }^{26}$ It has high interrater and test/retest reliability, internal consistency, adequate stability, ${ }^{27}$ and good construct and criterion validity. ${ }^{28}$ Items are scored on performance time and/or a 6-point ordinal scale measuring functional ability. The final score is the mean of individual task scores. ${ }^{26}$ This study used functional ability scores and not performance time scores. All primary outcomes were assessed by an experienced occupational therapist uninvolved with the arm training and unaware of participants' group assignment.

\section{Secondary outcome measure}

The Manipulation Check Questionnaire, based on a previous study, ${ }^{17}$ was administered every week (4 times total) to verify

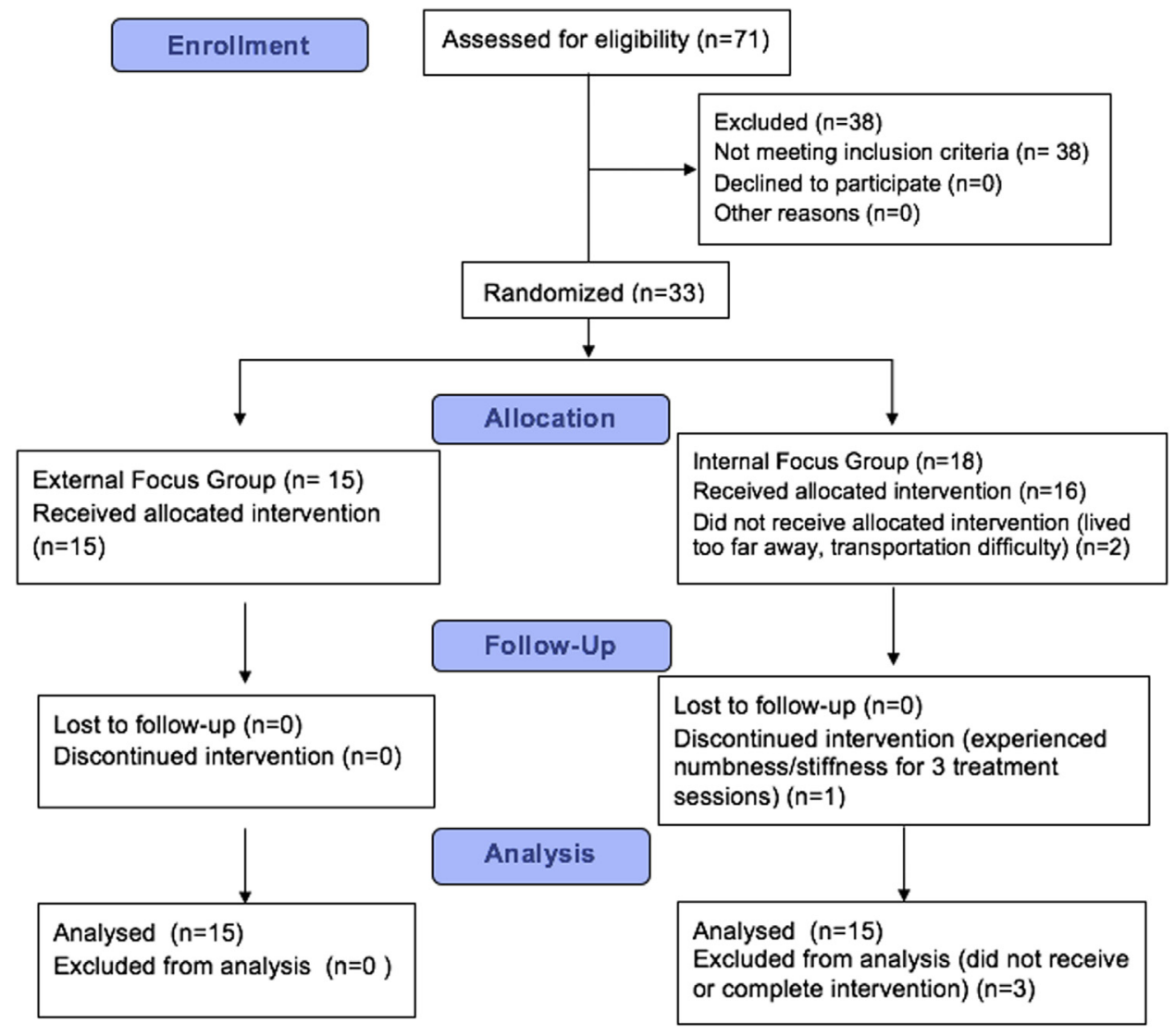

Fig 1 Consolidated Standards of Reporting Trials diagram—-participant recruitment and group assignment. 


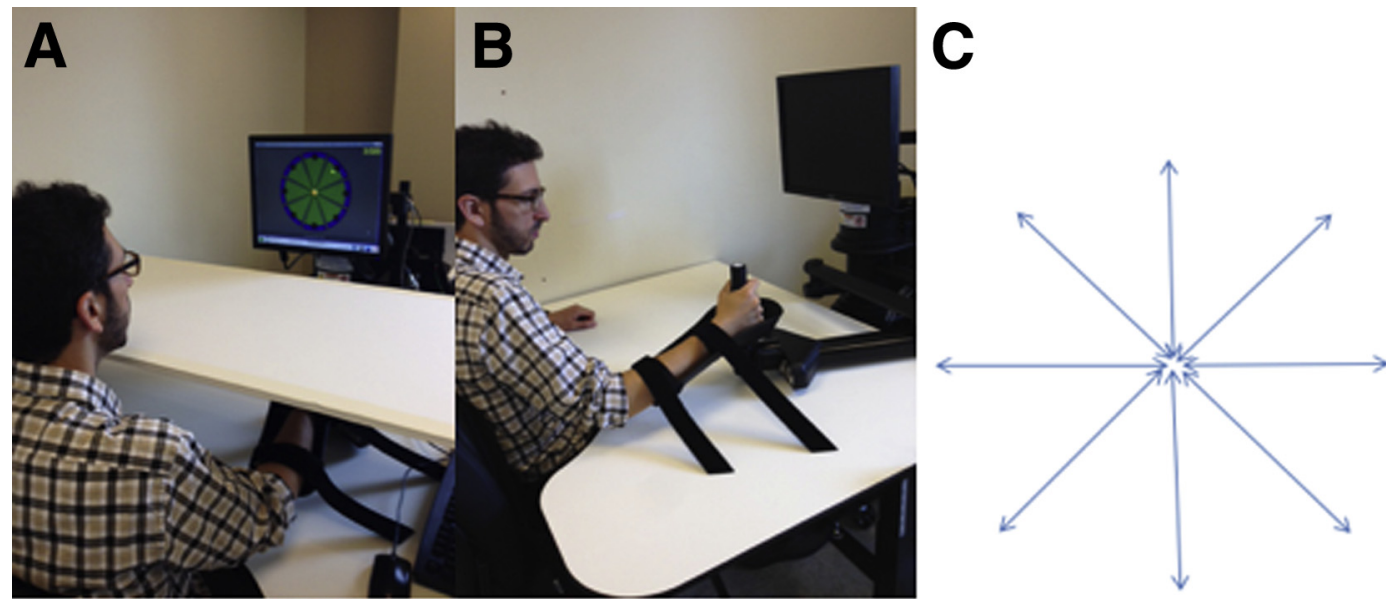

Fig 2 Practice conditions for the 2 treatment groups. (A) External focus group practice condition: video monitor on with arm occluded. Movement of the participant's arm was occluded from view to maximize attention on the video screen and the moving the yellow ball (task goal). (B) Internal focus group practice condition: video monitor off with arm not occluded. This practice condition maximized attention to movement of the arm, rather than an external task goal. (C) Eight-point clock pattern practiced by all participants during arm training. The internal focus group was oriented to the 8-point reaching pattern prior to the start of the treatment sessions and were directed to move their arm in this pattern during training. The external focus group practiced the identical 8-point clock pattern by moving the yellow ball target to the various targets on the video monitor. The arm training facilitated practice of shoulder flexion, extension, adduction, and abduction.

participants' degree of compliance with attentional focus cues during the study. The questionnaire was modified to include an initial open-ended question to minimize suggestive answers (appendix 2). Participants subsequently rated each of their open-ended answers on a 5-point Likert scale, ranging from 0 (none of the time) to 4 (all of the time). Study staff grouped participant responses into 3 categories: IF in nature, EF in nature, or other. Other was a catch-all category for responses that did not have EF or IF significance. Manipulation Check Questionnaire scores for each participant were determined by calculating the average score of each response category.

\section{Statistical analysis}

A power analysis was performed a priori using the $G^{*}$ Power (V3.1) software. ${ }^{29, \mathrm{c}} \mathrm{A}$ mixed, repeated-measures analysis of variance was selected with a medium effect size of .25 (based on previous robotics studies using the FMA as a primary outcome $^{30-32}$ ), power of $0.8, \alpha$ level of .05 (2-tailed), 2 groups, 3 measurements, a correlation among repeated measures of 0.5 , and nonsphericity correction equal to 1 . The total sample size was calculated as 28 . Thirty participants were estimated to adequately test the hypothesis.

\section{Results}

Sixteen women and 14 men with an average age of $58.1 \pm 12.6$ years and average time since a stroke of $5.08 \pm 3.44$ years completed the study. Thirty-three participants were enrolled in the study with 3 early withdrawals for a final sample size of 30 . Two participants withdrew because of transportation difficulties. One participant was withdrawn because of complaints of increased muscle stiffness and finger numbness during arm training. Session lengths ranged from 30 to 90 minutes based on the motor ability of the participant. The EF group completed on average more repetitions per treatment session (mean, 935.1) than the IF group (mean, 883.8). See table 1 for complete demographic data and table 2 for descriptive data of outcome measures.

There were no between-group differences for joint independence at discharge or follow-up, indicating no interaction effect for attentional focus between the 2 treatment groups. However, there were significant within-group differences for joint independence $\mathrm{EF}$ condition and joint independence IF condition $\left(\mathrm{F}_{1.6,45.4}=17.70 ; \quad P<.0005 ; \quad\right.$ partial $\quad \eta^{2}=.39 ; \quad \mathrm{F}_{2,56}=18.60 ;$ $P<.0005$; partial $\eta^{2}=.40$, respectively). Bonferroni-adjusted post hoc tests indicated significant improvement for joint independence EF condition and joint independence IF condition from baseline to discharge and baseline to follow-up $(P=.002, P<.0005 ; P=.001$, and $P<.0005$, respectively), but no significant difference from discharge to follow-up $(P=.461)$. See table 3 for details. Time accounted for $39 \%$ and $40 \%$ of the variance in change scores for joint independence EF condition and joint independence IF condition, respectively, controlling for attentional focus. There are no

Table 2 Scores for outcome measures at baseline, discharge, and 4-week follow-up $(n=30)$

\begin{tabular}{rlllll}
\hline Timepoint & Group & Jt-indep-EF & Jt-indep-IF FMA & \multicolumn{2}{l}{ WMFT } \\
\hline Baseline & EF & $.55 \pm .13$ & $.54 \pm .12$ & $19.63 \pm 12.62$ & $1.80 \pm .69$ \\
& IF & $.53 \pm .19$ & $.53 \pm .12$ & $17.80 \pm 8.92$ & $1.73 \pm .45$ \\
Discharge & EF & $.65 \pm .14$ & $.60 \pm .12$ & $23.13 \pm 12.87$ & $1.99 \pm .70$ \\
& IF & $.61 \pm .19$ & $.60 \pm .13$ & $21.73 \pm 9.67$ & $1.91 \pm .57$ \\
Follow-up & EF & $.69 \pm .13$ & $.62 \pm .11$ & $23.53 \pm 12.99$ & $2.03 \pm .72$ \\
& IF & $.62 \pm .16$ & $.62 \pm .12$ & $23.33 \pm 10.91$ & $2.00 \pm .59$ \\
\hline
\end{tabular}

NOTE. Values are mean \pm SD. There were no significant differences between the 2 treatment groups at baseline on all outcome measures. Maximum scores for the outcome measures: Jt-indep-EF $=1$, Jt-indep$\mathrm{IF}=1, \mathrm{FMA}=60$, and $\mathrm{WMFT}=5$.

Abbreviations: Jt-indep-EF, joint independence EF condition; Jt-indepIF, joint independence IF condition. 
Table 3 Analysis of variance significant main effects with post hoc testing

\begin{tabular}{|c|c|c|c|c|c|c|c|}
\hline & \multirow[b]{2}{*}{$\mathrm{F}$} & \multirow[b]{2}{*}{ Sig } & \multirow{2}{*}{$\begin{array}{l}\text { Partial } \\
\eta^{2}\end{array}$} & \multicolumn{4}{|c|}{ Post Hoc Testing } \\
\hline & & & & $1 \rightarrow 2 \Delta V$ & $2 \rightarrow 3$ & $\Delta V \quad 1 \rightarrow 3$ & $\Delta \mathrm{V}$ \\
\hline Jt-indep-EF* & 17.74 & $.000^{\dagger}$ & .39 & $.002^{\dagger}$ & .420 & $.02 .000^{\dagger}$ & .12 \\
\hline Jt-indep-IF & 18.60 & $.000^{\dagger}$ & .40 & $.001^{\dagger}$ & .470 & $.02 .000^{\dagger}$ & .09 \\
\hline FMA & 27.83 & $.000^{\dagger}$ & .50 & $.000^{\dagger} 3.72$ & .370 & $.96 .000^{\dagger}$ & 4.72 \\
\hline WMFT & 14.50 & $.000^{\dagger}$ & .36 & $.003^{\dagger} \quad .18$ & .419 & $.06 .000^{\dagger}$ & .24 \\
\hline
\end{tabular}

Abbreviations: $\Delta V$, change in absolute value of outcome variable; $1 \rightarrow 2, P$ value from baseline to discharge; $2 \rightarrow 3, P$ value from discharge to follow-up; $1 \rightarrow 3, P$ value from baseline to follow-up; Jtindep-EF, joint independence EF condition; Jt-indep-IF, joint independence IF condition; Sig, significance.

* Greenhouse-Geisser coefficient used to interpret within-subject effects for Jt-indep-EF because of significant Mauchly test.

${ }^{\dagger} P$ value significant at .05 level.

established minimal clinically important difference scores for joint independence; however, the relative changes from the total score for joint independence EF condition and joint independence IF condition were the following: $6.7 \%$ and $9.2 \%$ increase from baseline to discharge and $8.5 \%$ and $12 \%$ increase from baseline to follow-up, respectively. Figure 3 illustrates 4 total conditions: the relation between the 2 treatment groups across time under both the $\mathrm{EF}$ and IF practice conditions.

There were no between-group differences for the FMA and WMFT at discharge or follow-up, indicating no interaction effects of attentional focus (figs 4 and 5). Scores for the FMA and WMFT were significant within groups across time $\left(\mathrm{F}_{2,56}=27.83 ; P<.0005\right.$; partial $\eta^{2}=.50 ; \mathrm{F}_{2,56}=15.14 ; P<.0005$; partial $\eta^{2}=.35$, respectively). Bonferroni-adjusted post hoc tests indicated significant improvement from baseline to discharge and baseline to follow-up for the FMA and WMFT $(P<.0005, P<.0005 ; P=.002$, and $P<.0005$, respectively), with no significant differences from discharge to follow-up ( $P=.364$ and $P=.367$, respectively). See table 3 for details. Time accounted for $50 \%$ and $35 \%$ of the variance in change scores for the FMA and WMFT, respectively, controlling for attentional focus. Improvements did not reach minimal clinically important difference levels for both outcomes.

For the Manipulation Check Questionnaire, the EF group rated responses that were more externally focused in nature (mean, 3.17) than the IF group (mean, .85), and this was statistically significant $\left(t_{28}=8.75, P<.005\right)$. The IF group rated responses that were more internally focused in nature (mean, 3.21) than the EF group (mean, 1.41), and this was statistically significant

\section{Joint Independence}

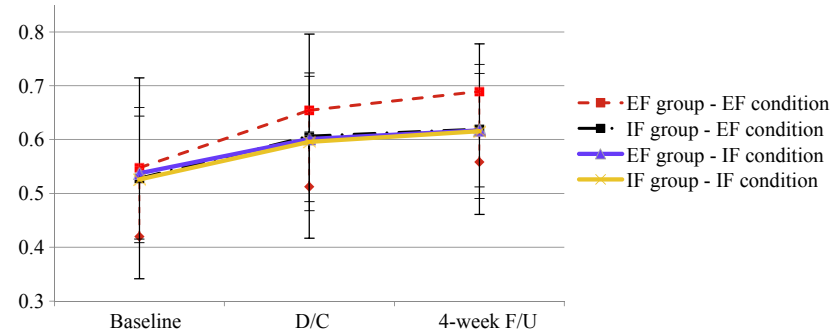

Fig 3 Joint independence assessed under EF and IF practice conditions for both groups. Abbreviations: D/C, discharge; F/U, followup.
FMA

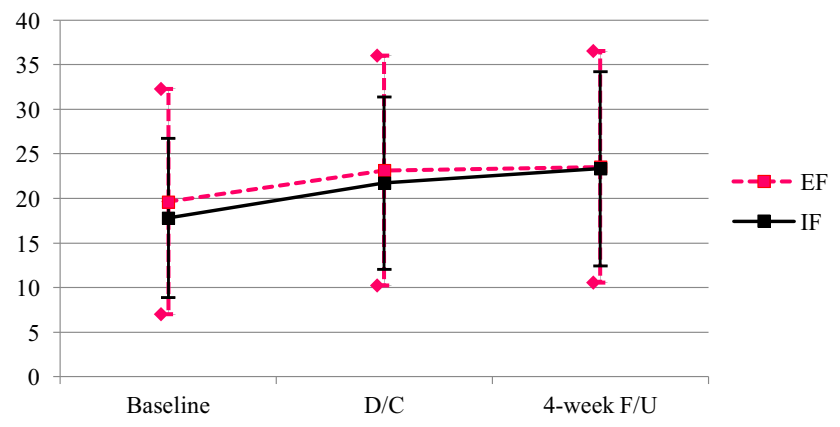

Fig 4 FMA scores for the 2 treatment groups at baseline, $D / C$, and F/U. Abbreviations: D/C, discharge; F/U, follow-up.

$\left(t_{28}=5.11, P<.005\right)$. Participants in both groups reported thinking about other things (eg, "my next meal," "what I have to do for the rest of the day," "am I doing this right?"). The EF group had an average score of .72, and the IF group had an average score of 1.07. This difference was not statistically significant.

\section{Discussion}

To our knowledge, this is the first study to investigate the effects of EF versus IF after 12 sessions of arm training in individuals with severe and moderate arm impairment after chronic stroke. We hypothesized that the EF group would have greater retention in joint independence, FMA, and WMFT at follow-up compared with the IF group. The study results did not support our hypotheses. There was no differential effect of attentional focus for participants at discharge or 4 weeks after training. Regardless of attentional focus, all participants improved at the same rate and maintained the improvements for at least 4 weeks. Manipulation Check Questionnaire results demonstrated that participants were mostly compliant with their assigned attentional focus, and thought about other things at a lower frequency.

Our results were consistent with a recent study by Kal et al, ${ }^{19}$ suggesting that individuals with stroke may not learn motor skills in the same way as healthy populations. Our results contradicted much of the established literature regarding the healthy population and the constrained action hypothesis, which favors EF over IF during motor skill training. ${ }^{12,33-39}$ According to the constrained action hypothesis, EF elicits better performance in healthy individuals because it increases the coherence of sensory input and effector output, improving the coupling of the agonist/antagonist

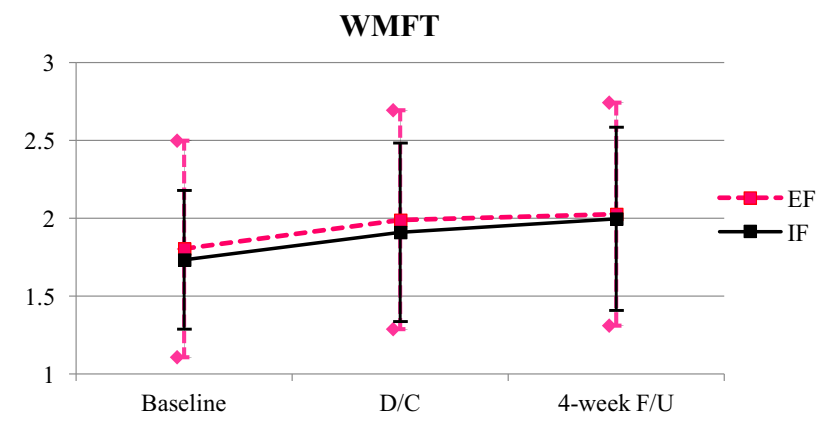

Fig 5 WMFT scores for the 2 treatment groups at baseline, $D / C$, and F/U. Abbreviations: D/C, discharge; F/U, follow-up. 
muscles; IF interrupts the sensory input and constrains the effector output hindering motor coordination. ${ }^{6}$

The presence of disease or age can disrupt and compromise this sensorimotor integration. ${ }^{40,41}$ Individuals with moderate-tosevere arm impairment after stroke experience a disruption in the effector output associated with hemiparesis of the affected arm. Weakness in the muscles of the shoulder, elbow, wrist, and hand is common for people with moderate-to-severe motor impairments. This pattern of hemiparesis contributes to difficulties with active movement and motor control of movement patterns (eg, forward reach, elbow extension, wrist/finger extension).

Based on the Manipulation Check Questionnaire, 33\% of participants in the EF group reported switching from EF to IF when practicing reaching patterns that were difficult to achieve. They specifically thought about straightening their elbow when attempting to increase horizontal shoulder flexion and horizontal shoulder abduction. It is possible that participants with moderateto-severe arm impairment may benefit from adopting an IF when practicing challenging motor skills to facilitate improved effector output. This could be a possible explanation for why we did not detect an advantage for adopting an EF during training.

Another possible explanation comes from recent studies in stroke rehabilitation supporting the idea that regardless of intervention type, participants tend to achieve similar gains in upper and lower limb training if the interventions are dose-matched. ${ }^{42-44}$ It is worth noting that the participants in the EF group completed more repetitions per session (mean, 935.1) than the IF group (mean, 883.8). Reasons for reduced dosage for both groups included arriving late/transportation issues and complaints of fatigue (especially during early sessions; this improved over time as subjects got used to the protocol). It is possible that the IF group was more prone to fatigue than the EF group because the EF practice conditions were more dynamic. However, the similar rate of improvements in both groups suggests that the dosage difference did not affect results. The relatively comparable amount of repetitions practiced per treatment group may be the best explanation for why both groups improved in the same manner and suggests that the amount of practice was more determinant of motor learning and retention than the type of attentional focus used during training.

\section{Study limitations}

A primary limitation for studies involving attentional focus is the lack of an objective measure to determine what someone is paying attention to while performing a motor task. We used a qualitative questionnaire to address this limitation. Interestingly, during the training period, both groups reported thinking about a combination of EF and IF aspects of the task, and many of the participants thought about unrelated things (eg, their schedule, their next meal). The results from the Manipulation Check Questionnaire demonstrate that strict compliance to one type of attentional focus may be difficult to achieve in studies that require an extended training period with a significant number of sessions. Detectable changes between the EF and IF groups may have been limited because standardized robotics training provided all participants haptic and performance feedback throughout the training. The additional feedback may have diminished the effects of attentional focus. Additionally, our selected treatment did not yield clinically significant improvements overall. A training approach with more robust effectiveness may produce more definitive results when comparing EF and IF groups. The results apply to individuals with moderate-to-severe upper limb impairment. It is unknown how attentional focus affects the retention of motor improvements in individuals in the acute phase of stroke, lower limb impairment, or mild upper limb impairment.

Wulf and Lewthwaite ${ }^{7}$ recently introduced a new theory called Optimizing Performance Through Intrinsic Motivation and Attention for Learning, which describes motor behavior within the context of the learner's motivation and attention. The authors have expanded on the constrained action hypothesis by adding intrinsic motivation and its underlying components along with attentional focus as contributing factors for acquiring new motor skills. ${ }^{7}$ The components of intrinsic motivation were not addressed or controlled for in our study; therefore, we cannot comment on how motivation may or may not have influenced our results.

\section{Directions for future research}

Subsequent studies should investigate the effects of attentional focus on the retention of motor improvement for individuals with mild arm impairment and lower limb impairment after stroke. A treatment approach with robust clinical effectiveness should be selected as the underlying motor training protocol.

\section{Conclusions}

There were no differences in retention of motor skills between EF and IF participants 4 weeks after arm training, suggesting that individuals with moderate-to-severe arm impairment may not experience the advantages of EF found in healthy individuals. Attentional focus is most likely not an active ingredient for retention of trained motor skills for individuals with moderate-to-severe arm impairment, whereas dosage and intensity of practice appear to be pivotal.

\section{Suppliers}

a. SPSS Version 23; IBM.

b. InMotion ARM; Bionik.

c. G*Power Version 3.1; Heinrich Heine Universitat Dusseldorf.

\section{Keywords}

Attention; Rehabilitation; Stroke; Upper extremity; Robotics

\section{Corresponding author}

Grace J. Kim, PhD, OTR/L, Department of Occupational Therapy, Steinhardt School Culture, Education, and Human Development, New York University, Pless Hall, 82 Washington Square E, 6th Fl, New York, NY 10003. E-mail address: gjk207@ nyu.edu.

\section{Acknowledgments}

We thank Sharon L. Weinberg, $\mathrm{PhD}$, for her assistance with study design and data analysis; and Terrence Hicks, MS, OTR/L, for his contribution to data collection. 


\section{Appendix 1 Inclusion/Exclusion Criteria and Recruitment Methods}

Inclusion criteria were as follows: diagnosis of stroke $\geq 6$ months; moderate-to-severe arm impairment (defined as FMA scores between 1 and 19 for severe and 20 and 47 for moderate $^{18}$ ); intact visual scanning and spatial orientation of the affected arm; functional cognitive status; and participants were medically stable. Primary exclusions included the following: concurrent occupational or physical therapy for the arm; joint contractures at the wrist, forearm, elbow, or shoulder; moderate-to-severe spasticity at the elbow or shoulder (modified Ashworth scale score $>2$ ); presence of other neurologic conditions; botulinum toxin injection in the affected arm $<3$ months prior; and score of 0 of 5 on manual muscle test at the elbow or shoulder.

Starting in July 2014, participants were recruited through a hospital-based stroke research registry, referrals from outpatient physicians and therapists, outreach to community stroke support groups/organizations, and study flyers in health care settings. Data were collected between September 2014 through November 2015.

\section{Appendix 2 Manipulation Check Questionnaire}

Since the last time I asked, what were you thinking about while arm training on the robot? (multiple answers ok, include subject ratings after each response)

5-point Likert scale to rate answers

$0=$ None of the time

$1=\mathrm{A}$ little of the time

$2=$ Some of the time

$3=$ Most of the time

$4=$ All of the time

Notes/comments

NOTE. Adapted with permission from Fasoli et al. ${ }^{17}$

\section{References}

1. Kahneman D. Attention and effort. New Jersey: Prentice Hall; 1973.

2. Wulf G. Attention and motor skill learning. Champaign: Human Kinetics; 2007.

3. Magill R. Motor learning and control: concepts and application. 9th ed. New York: McGraw Hill; 2011.
4. Chiviacowsky S, Wulf G, Avila LT. An external focus of attention enhances motor learning in children with intellectual disabilities. J Intellect Disabil Res 2013;57:627-34.

5. Wulf G, McNevin N, Shea CH. The automaticity of complex motor skill learning as a function of attentional focus. Q J Exp Psychol A 2001;54:1143-54.

6. McNevin NH, Shea $\mathrm{CH}$, Wulf G. Increasing the distance of an external focus of attention enhances learning. Psychol Res 2003;67:22-9.

7. Wulf G, Lewthwaite R. Optimizing performance through intrinsic motivation and attention for learning: the OPTIMAL theory of motor learning. Psychon Bull Rev 2016;23:1382-414.

8. Gokeler A, Benjaminse A, Welling W, Alferink M, Eppinga P, Otten B. The effects of attentional focus on jump performance and knee joint kinematics in patients after ACL reconstruction. Phys Ther Sport 2015;16:114-20.

9. Kal EC, van der Kamp J, Houdijk H. External attentional focus enhances movement automatization: a comprehensive test of the constrained action hypothesis. Hum Mov Sci 2013;32:527-39.

10. Lohse KR, Sherwood DE, Healy AF. On the advantage of an external focus of attention: a benefit to learning or performance? Hum Mov Sci 2014;33:120-34.

11. McNevin NH, Wulf G, Carlson C. Effects of attentional focus, selfcontrol, and dyad training on motor learning: implications for physical rehabilitation. Phys Ther 2000;80:373-85.

12. Shea $\mathrm{CH}$, Wulf G. Enhancing motor learning through external-focus instructions and feedback. Hum Mov Sci 1999;18:553-71.

13. Welling W, Benjaminse A, Gokeler A, Otten B. Enhanced retention of drop vertical jump landing technique: a randomized controlled trial. Hum Mov Sci 2016;45:84-95.

14. Wulf G. Attentional focus and motor learning: a review of 10 years of research. E-Journal Bewegung und Training 2007;1:1-11.

15. Wulf G, Prinz W. Directing attention to movement effects enhances learning: a review. Psychon Bull Rev 2001;8:648-60.

16. Wulf G, Shea C, Park JH. Attention and motor performance: preferences for and advantages of an external focus. Res Q Exerc Sport 2001;72:335-44.

17. Fasoli SE, Trombly CA, Tickle-Degnen L, Verfaellie MH. Effect of instructions on functional reach in persons with and without cerebrovascular accident. Am J Occup Ther 2002;56:380-90.

18. Durham KF, Sackley CM, Wright CC, Wing AM, Edwards MG, van Vliet P. Attentional focus of feedback for improving performance of reachto-grasp after stroke: a randomised crossover study. Physiotherapy 2014; $100: 108-15$.

19. Kal E, van der Kamp J, Houdijk H, Groet E, van Bennekom C, Scherder E. Stay focused! The effects of internal and external focus of attention on movement automaticity in patients with stroke. PLoS One 2015;10:e136917.

20. Balasubramanian S, Colombo R, Sterpi I, Sanguineti V, Burdet E. Robotic assessment of upper limb motor function after stroke. Am J Phys Med Rehabil 2012;91(11 Suppl 3):S255-69.

21. Bosecker C, Dipietro L, Volpe B, Krebs HI. Kinematic robot-based evaluation scales and clinical counterparts to measure upper limb motor performance in patients with chronic stroke. Neurorehabil Neural Repair 2010;24:62-9.

22. Dipietro L, Krebs HI, Fasoli SE, et al. Changing motor synergies in chronic stroke. J Neurophysiol 2007;98:757-68.

23. Gladstone DJ, Danells CJ, Black SE. The fugl-meyer assessment of motor recovery after stroke: a critical review of its measurement properties. Neurorehabil Neural Repair 2002;16:232-40.

24. See J, Dodakian L, Chou C, et al. A standardized approach to the fuglmeyer assessment and its implications for clinical trials. Neurorehabil Neural Repair 2013;27:732-41.

25. Woodbury ML, Velozo CA, Richards LG, Duncan PW. Rasch analysis staging methodology to classify upper extremity movement impairment after stroke. Arch Phys Med Rehabil 2013;94:1527-33.

26. Woodbury M, Velozo CA, Thompson PA, et al. Measurement structure of the Wolf Motor Function Test: implications for motor control theory. Neurorehabil Neural Repair 2010;24:791-801. 
27. Morris DM, Uswatte G, Crago JE, Cook EW 3rd, Taub E. The reliability of the Wolf Motor Function Test for assessing upper extremity function after stroke. Arch Phys Med Rehabil 2001;82:750-5.

28. Wolf SL, Catlin PA, Ellis M, Archer AL, Morgan B, Piacentino A. Assessing Wolf motor function test as outcome measure for research in patients after stroke. Stroke 2001;32:1635-9.

29. Faul F, Erdfelder E, Lang AG, Buchner A. G*Power 3: a flexible stastistical power and analysis program for the social, behavioral, and biomedical sciences. Behav Res Methods 2007;39:175-91.

30. Fasoli SE, Krebs HI, Stein J, Frontera WR, Hughes R, Hogan N. Robotic therapy for chronic motor impairments after stroke: follow-up results. Arch Phys Med Rehabil 2004;85:1106-11.

31. Krebs HI, Volpe BT, Williams D, et al. Robot-aided neurorehabilitation: a robot for wrist rehabilitation. IEEE Trans Neural Syst Rehabil Eng 2007;15:327-35.

32. Volpe B, Lynch D, Rykman-Berland A, et al. Intensive sensorimotor arm training mediated by therapist or robot improves hemiparesis in patients with chronic stroke. Neurorehabil Neural Repair 2008;22:305-10.

33. Landers M, Wulf G, Wallmann H, Guadagnoli M. An external focus of attention attenuates balance impairment in patients with Parkinson's disease who have a fall history. Physiotherapy 2005;91:152-8.

34. Wulf G. Attentional focus effects in balance acrobats. Res Q Exerc Sport 2008;79:319-25.

35. Wulf G, Landers M, Lewthwaite R, Tollner T. External focus instructions reduce postural instability in individuals with Parkinson disease. Phys Ther 2009;89:162-8.
36. Wulf G, Lauterbach B, Toole T. The learning advantages of an external focus of attention in golf. Res Q Exerc Sport 1999;70: 120-6.

37. Wulf G, McConnel N, Gartner M, Schwarz A. Enhancing the learning of sport skills through external-focus feedback. J Mot Behav 2002;34: $171-82$.

38. Wulf G, Su J. An external focus of attention enhances golf shot accuracy in beginners and experts. Res Q Exerc Sport 2007;78:384-9.

39. Zachry T, Wulf G, Mercer J, Bezodis N. Increased movement accuracy and reduced EMG activity as the result of adopting an external focus of attention. Brain Res Bull 2005;67:304-9.

40. Gantert C, Honerkamp J, Timmer J. Analyzing the dynamics of hand tremor time series. Biol Cybern 1992;66:479-84.

41. Newell KM, Gao F, Sprague RL. The dynamical structure of tremor in tardive dyskinesia. Chaos 1995;5:43.

42. McCabe J, Monkiewicz M, Holcomb J, Pundik S, Daly JJ. Comparison of robotics, functional electrical stimulation, and motor learning methods for treatment of persistent upper extremity dysfunction after stroke: a randomized controlled trial. Arch Phys Med Rehabil 2015; 96:981-90.

43. Winstein CJ, Wolf SL, Dromerick AW, et al. Effect of a task-oriented rehabilitation program on upper extremity recovery following motor stroke: the ICARE randomized clinical trial. JAMA 2016;315:571-81.

44. Duncan PW, Sullivan KJ, Behrman AL, et al. Body-weightsupported treadmill rehabilitation after stroke. N Engl J Med 2011; 364:2026-36. 